\title{
SArdinia Roach2-based Digital Architecture for Radio Astronomy (SARDARA)
}

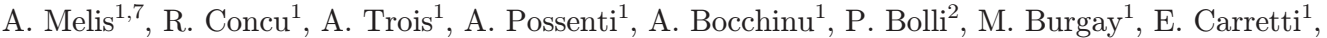 \\ P. Castangia ${ }^{1}$, S. Casu ${ }^{1}$, C. Cecchi Pestellini ${ }^{3}$, A. Corongiu ${ }^{1}$, N. D'Amico ${ }^{1}$, E. Egron ${ }^{1}$, \\ F. Govoni ${ }^{1}$, M. N. Iacolina ${ }^{6}$, M. Murgia ${ }^{1}$, A. Pellizzoni ${ }^{1}$, D. Perrodin ${ }^{1}$, M. Pilia ${ }^{1}$, \\ T. Pisanu ${ }^{1}$, A. Poddighe ${ }^{1}$, S. Poppi $^{1}$, I. Porceddu ${ }^{1}$, A. Tarchi ${ }^{1}$, V. Vacca ${ }^{1}$, \\ G. Aresu ${ }^{1}$, M. Bachetti ${ }^{1}$, M. Barbaro ${ }^{4}$, A. Casula ${ }^{4}$, A. Ladu ${ }^{1}$, S. Leurini ${ }^{1,5}$,

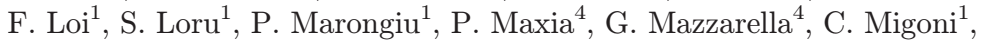 \\ G. Montisci ${ }^{1,4}$, G. Valente ${ }^{6}$ and G. Vargiu ${ }^{1}$ \\ ${ }^{1}$ INAF - Osservatorio Astronomico di Cagliari \\ Via della Scienza 5, 09047 Selargius, Italy \\ ${ }^{2}$ INAF - Osservatorio Astrofisico di Arcetri \\ Largo Enrico Fermi 5, I-50125 Firenze, Italy \\ ${ }^{3}$ INAF - Osservatorio Astronomico di Palermo \\ P.zza Parlamento 1, 90134 Palermo, Italy \\ ${ }^{4}$ DIEE University of Cagliari \\ Via Marengo 2, 09123 Cagliari, Italy \\ ${ }^{5}$ Max Planck Institut für RadioAstronomie \\ Auf dem Hügel 69, 53121 Bonn, Germany \\ ${ }^{6}$ Agenzia Spaziale Italiana \\ Via del Politecnico sn, 00133 Roma, Italy \\ 7amelis@oa-cagliari.inaf.it
}

Received 2017 September 11; Accepted 2018 March 12; Published 2018 April 2

The Sardinia Radio Telescope (SRT) is a 64-m, fully-steerable single-dish radio telescope that was recently commissioned both technically and scientifically with regard to the basic observing modes. In order to improve the scientific capability and cover all the requirements for an advanced single-dish radio telescope, we developed the SArdinia Roach2-based Digital Architecture for Radio Astronomy (SARDARA), a wideband, multi-feed, general-purpose, and reconfigurable digital platform, whose preliminary setup was used in the early science program of the SRT in 2016. In this paper, we describe the backend both in terms of its scientific motivation and technical design, how it has been interfaced with the telescope environment during its development and, finally, its scientific commissioning in different observing modes with singlefeed receivers.

Keywords: Backends, radio telescopes, single-dish.

This is an Open Access article published by World Scientific Publishing Company. It is distributed under the terms of the Creative Commons Attribution 4.0 (CC-BY) License. Further distribution of this work is permitted, provided the original work is properly cited. 


\section{Introduction}

The National Institute for Astrophysics $\left(\operatorname{INAF}^{(a)}\right)$, the major Italian research organization for astrophysics, operates three radio telescopes. Two are $32-\mathrm{m}$ across and are located in Medicina ${ }^{(\mathrm{b})}$ (Bologna) and Noto $^{(\mathrm{c})}$ (Siracusa), respectively. The third and largest one is the 64-m Sardinia Radio Telescope ${ }^{(\mathrm{d})}$ (SRT), located in San Basilio (Cagliari). Figure 1 shows their positions.

The Italian antennas have been equipped with several receivers, both single-feed and arrays, and span a broad frequency range (Valente et al., 2016). Currently, the SRT seven-beam K-band array (18-26.5 GHz; Orfei et al. (2010)) has the largest number of feeds and an intermediate frequency (IF) band of $0.1-2.1 \mathrm{GHz}$ for both polarizations. At the SRT, the ideal digital backend is thus expected to meet the following specifications:

- up to 14 IF signals simultaneously;

- up to $2.1 \mathrm{GHz}$ of bandwidth each;

- high flexibility for all scientific requirements of a single-dish radio telescope.

The three Italian radio telescopes have been equipped with a variety of backends (Melis et al., 2014),

\footnotetext{
${ }^{\mathrm{a}}$ http://www.inaf.it/en.

${ }^{\mathrm{b}}$ http://www.med.ira.inaf.it/.

${ }^{\mathrm{c}}$ http://www.noto.ira.inaf.it/.

${ }^{\mathrm{d}}$ http://www.srt.inaf.it/.
}

each of which is dedicated to a specific observing mode: Total Power (available at all three antennas), XARCOS (available at SRT and Medicina) and the Digital Filter Bank 3 (available at SRT). The Total Power (TP; Scalambra et al. (2013)) backend can use the entire available bandwidth $(2.1 \mathrm{GHz})$ and all $14 \mathrm{IF}$ signals ( 7 beams, dual polarization), but solely for continuum studies with neither spectral (just one broad frequency channel) nor full Stokes information; it offers a fast minimum sampling time of $1 \mathrm{~ms}$. XARCOS (Comoretto et al., 2006) is a spectrometer that records full-Stokes information for all 14 IFs, but with a narrow bandwidth (up to $125 \mathrm{MHz}$ ) and a long minimum sampling time $(10 \mathrm{~s})$, which makes it unsuitable for on-the-fly (OTF) mapping. Finally, the Digital Filter Bank 3 (DFB3; Egron et al. (2015)) can deliver a bandwidth of up to $1 \mathrm{GHz}$, but with only four IFs; it is unable to manage array systems with more than two beams.

In order to offer a backend that can manage all observing modes, that can cover the entire IF bandwidth, manage all $14 \mathrm{IFs}$, and operate with fast sampling times, we developed the SArdinia Roach2based Digital Architecture for Radio Astronomy (SARDARA) backend. This backend system is based on the new ROACH2 ${ }^{(\mathrm{e})}$ boards that were developed by the Collaboration for Astronomy

$\overline{{ }^{\mathrm{e}} \text { https://casper.berkeley.edu/wiki/ROACH-2_Revision_2. }}$

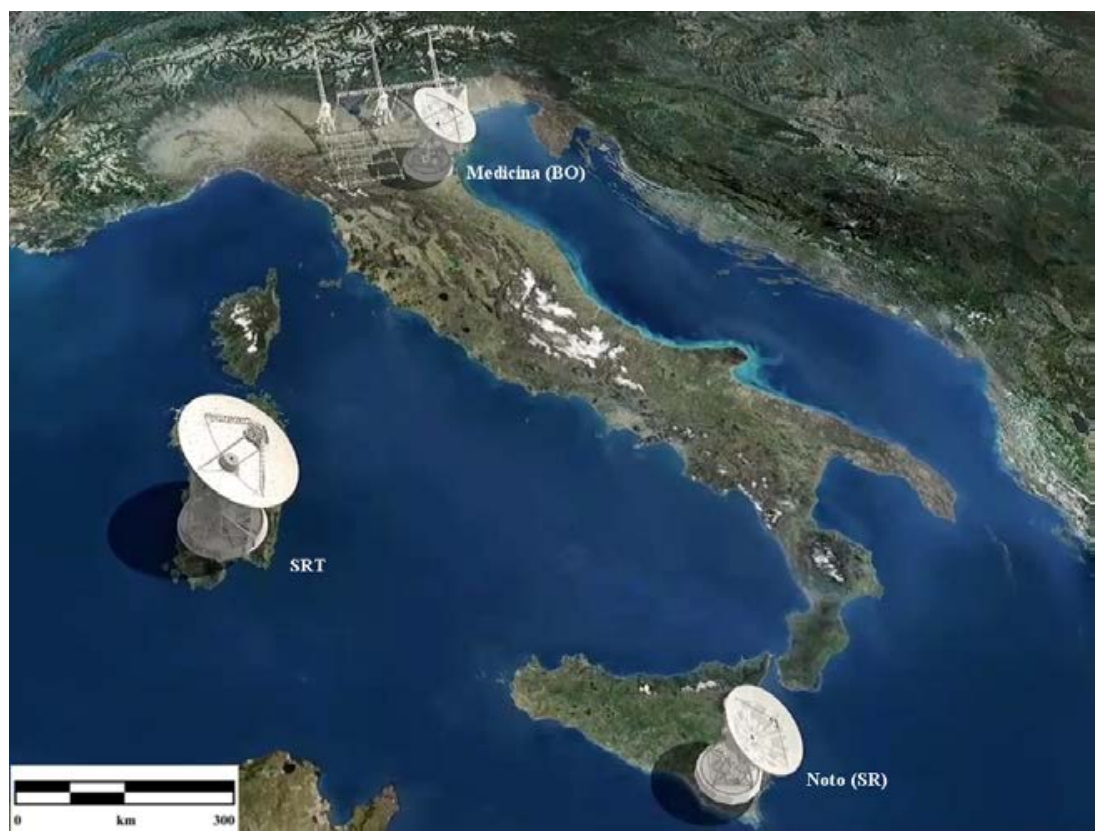

Fig. 1. Radio telescopes in Italy. 
Signal Processing and Electronic Research consortium (CASPER). ${ }^{(\mathrm{f})}$ The core of the boards is made of a Xilinx Virtex 6 Field Programmable Gate Array (FPGA), and their 5 GSample/s ADCs are able to digitize frequency bands of up to $2.5 \mathrm{GHz}$, which is sufficient for our needs.

The paper is arranged as follows: after a detailed description of SARDARA (Sec. 2), we present how it has been interfaced with the telescope control software during development (Sec. 3). In Sec. 4, we present the scientific validation - for single-feed receivers - of a diverse set of observing modes developed for SARDARA, together with a comparison of the performances obtained at other facilities. Finally, we report our conclusions in Sec. 5.

\section{The SARDARA Backend}

In this section, we describe the SARDARA backend, both in terms of its scientific motivations and technical design.

\subsection{Scientific motivations}

The SRT is a very versatile instrument; it should therefore be provided with a similar flexible digital backend, i.e. a fully-reconfigurable architecture capable of covering as many observing modes of a single-dish radio telescope as possible: continuum, polarimetry, spectroscopy, high-time resolution for pulsars and fast transients. As mentioned in Sec. 1, the telescope is equipped with a variety of digital platforms that we briefly described. Although all of them adopt reconfigurable hardware like FPGAs, they were designed and built for a specific purpose, and could not easily be upgraded for other purposes. Therefore, we decided to develop a whole new platform as the main backend for all observing modes, which in particular could fully exploit the seven-feed K-band receiver.

We chose to use, as the centerpiece, the ROACH2 stand-alone FPGA-based boards, one for each of the seven feeds. Each board is also equipped with two 5 GSample/s analog-to-digital conversions $(\mathrm{ADCs})^{(\mathrm{g})}$ as well as with two mezzanine cards ${ }^{(\mathrm{h})}$ able to manage up to $40 \mathrm{Gbps}$ each. ROACH2 boards were also chosen for the following reasons: reconfigurability, ability to cover an instantaneous

\footnotetext{
f ${ }_{\text {https: / / casper.berkeley.edu/. }}$

${ }^{g}$ https://casper.berkeley.edu/wiki/ADC1x5000-8.

${ }^{\mathrm{h}}$ https://casper.berkeley.edu/wiki/SFP\%2B.
}

bandwidth of up to $2.5 \mathrm{GHz}$, a data rate of up to $80 \mathrm{Gbps}$ (both in input and output). In order to manage the data and to post-process them if required, we also need a set of workstations. In principle, after the ADC and the digital signal processing, each $\mathrm{ROACH} 2$ can provide the digital data via eight $10 \mathrm{Gbit} / \mathrm{s}$ links, i.e. the eight outputs of its two mezzanine cards. This would allow us to send out the entire raw data provided by the two ADCs (each sample is represented with 8 bits, so each of the two ADCs produces up to $40 \mathrm{Gbps}$ ); in this case, the $\mathrm{ROACH} 2$ only operates as a digitizer and packetizer. In order to handle such a huge rate of data for all ROACH2s, we would need a switch with a considerable number of ports, as well as a number of workstations capable of managing all these data streams. This would be a problem, both technically and logistically as well as in terms of very high cost.

However, the requirements in terms of data processing can often be satisfied by the ROACH2s themselves, allowing us to perform wide-band (WB) full-Stokes spectrometers (see Table A.1 in the appendix) with a considerable (up to 16,384) number of channels. Usually, this configuration is suitable for most scientific applications; for example, all experimental results (regarding continuum, polarimetry, spectroscopy and high-time resolution observations) shown in Sec. 4 were obtained with data processed on FPGAs only. Moreover, since each spectrum is integrated in time, the output data rate (of the order of a few hundreds of Mbit/sec) was totally manageable with a single 10 Gbps link. Indeed, one of the eight ports of the mezzanine cards is often enough; therefore for these applications, we need seven workstations (one for each ROACH2).

There are also astronomical studies where the WB raw data recording and/or post-processing can be needed, in particular for pulsar timing and highfrequency resolution spectroscopy. Pulsar timing is typically performed with single-feed observations. The signal can then be split into a certain number of sub-bands. For this purpose, up to eight of the 10 Gbps links of one ROACH2 - the one at which the single-feed is fed - should be made available. Once these baseband data are provided to workstations, online dedispersion and folding (see Table A.2 in the appendix) can be applied at the known dispersion measure (DM) and using the pulsar's rotational ephemeris. Therefore, for this application, we need up to eight workstations (one for each sub-band provided by a single ROACH2). 
Even for WB high-frequency resolution spectroscopy, since 16,384 channels are considerably lower than those requested, we need baseband raw data; what is different is the post-processing task, which consists in a further channelization in order to achieve a high-frequency resolution - of the order of up to a few hundreds of $\mathrm{Hz}$ - needed for studying narrow-band (NB) emissions (see Table A.3 in the appendix).

Unlike for pulsar timing, spectroscopy might take advantage of a multi-feed configuration, for spectroscopic mapping and spectral line searches over a large bandwidth in spatially-extended targets. For known lines, the so-called "zoom" modes (see Table A.4 in the appendix) can be used to select and send out (from the ROACH2) only the portions of the bandwidth around those NB lines, by allowing the use of only a single 10 Gbps output for each board. Once the data are provided, a polyphase filter bank ${ }^{(\mathrm{i})}(\mathrm{PFB})$ with a large number of channels is performed for each portion of the bandwidth. Seven workstations (one for each $\mathrm{ROACH} 2$ ) are sufficient in this case as well.

For Galactic or extragalactic spectroscopic surveys, where NB emissions are unknown, both in frequency position and width, the WB multi-feed high-frequency resolution spectrometers would be extremely helpful; however, this is unsuitable unless one addresses the aforementioned issues (we would need, for example, 56 workstations). As a consequence, for the latter as well as for multi-feed highfrequency resolution spectroscopic images, astronomers have to settle for a reduced bandwidth (see Table A.4 in the appendix), which can be handled with the seven workstations that have been repeatedly mentioned.

Given the aforementioned discussion, we developed the infrastructure described in the following section.

\subsection{Technical design}

In this section, we describe the technical design of the SARDARA backend. Figure 2 shows the entire infrastructure, built by taking into consideration the technical needs that were described in the previous section.

The backend is composed of seven ROACH2s, eight workstations, a switch, a data storage, a clock distributor, a pulse-per-second (PPS) distributor,

\footnotetext{
${ }^{\mathrm{i}}$ https://casper.berkeley.edu/wiki/The_Polyphase_Filter_ Bank_Technique.
}

and a control computer that communicates with the antenna control software. All modules are connected via a 10 Gbps optical fiber, indicated with blue arrows; we note that each element of the backend can be reached by each other and, vice versa, each element can reach each other. This allows us to distribute the computing among nodes quite easily. A 1 Gbps connection is also available (we did not draw these connections for keeping the diagram clearer, as well as for the clock and PPS signals) only to control each element of the backend.

Since we have decided to have eight workstations, a 24-port switch is suitable for SARDARA as shown in Fig. 2; we have chosen the Brocade VDX 6720, one of the best systems on the market at the time of purchase.

The processing units (indicated with "GPUbased node" in Fig. 2) are very powerful computers, both in terms of handling the high-speed data and of real-time post-processing, as well as of data storing capability. We chose the motherboard Supermicro X9DRI-F equipped with two Intel XEON 8 core E5-2640 v2, while the chassis is a Supermicro $4 \mathrm{U}$ E-ATX CSE-745TQ-R1200B. Table 1 shows the main characteristics of each computing node.

The Graphical Processing Units (GPUs) are high-performance units that can be used to postprocess the raw data, so we will use them for the applications mentioned in the previous section (pulsar timing and high-frequency resolution spectroscopy). Although they suffer intrinsic limits of the Von Neumann architecture, once the data are available, GPUs represent one of the best solutions, especially for parallel computing. At the time of purchase, the hardware, the Nvidia GTX 980 TI was the best choice in terms of price performance; each node is equipped with two of these GPUs. The available 6 GB RAM memory and the 2816 cores allow for a real-time FFT engine to be applied. We have performed some tests on base-band data (bandwidth $150 \mathrm{MHz}$ ) provided by a $\mathrm{ROACH} 2$ : by applying a PFB with four taps we obtained, in realtime, up to one million of spectral channels. This is largely sufficient for spectroscopic observations at high-frequency resolution.

Another major piece of hardware for SARDARA is the data storage. Usually, when a data acquisition is performed, we should avoid any overload of the workstations, in order to prevent packet loss or similar issues. On the other hand, the observer may need to retrieve the data as soon as possible, for instance, in order to do preliminary 


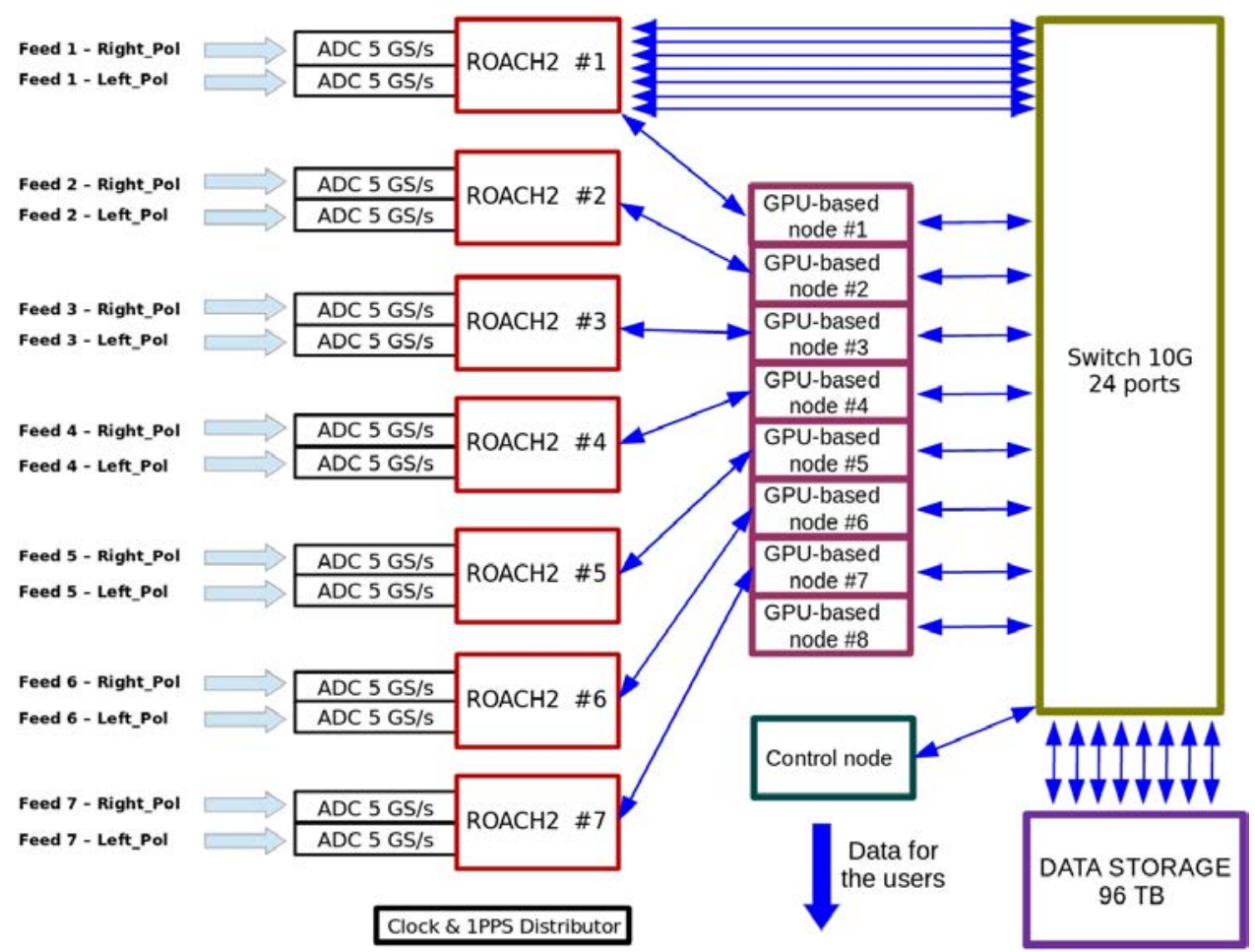

Fig. 2. SARDARA block diagram. Blue arrows represent 10 Gbps optical fiber links.

Table 1. SARDARA computing node.

\begin{tabular}{ll}
\hline Chassis & Supermicro 4U E-ATX CSE-745TQ-R1200B \\
Motherboard & Supermicro X9DRI-F \\
CPU & 2 Intel XEON 8 core E5-2640 v2 \\
GPU & 2 NVidia GTX 980 TI \\
NIC & Intel X520 $2 \times 10$ Gbps \\
Memory & 256 GB DDR3 \\
Hard Disk & 4 Western Digital 4 TB \\
\hline
\end{tabular}

data reduction and verify the data quality. In this regard, the adopted solution was to employ a data storage unit with large capacities in terms of data transfer and efficiency; thus, we opted for the Fujitsu ETHERNUS DX80/90 S2. ${ }^{(\mathrm{j})}$

The system has eight $10 \mathrm{Gbit} / \mathrm{s}$ iSCSI interfaces, which are all connected to the Brocade switch in order to fully exploit the input/output capability of the system. The modular, rack mounted storage consists of two controllers that are used to deal with the writing/reading requests via the $10 \mathrm{Gbit} / \mathrm{s}$ iSCSI interfaces. The controllers can manage up to 24 disks in several RAID levels; we opted for a RAID 0 configuration. Indeed, with the latter, we can achieve high read/write performance and $100 \%$ disk utilization; moreover, since the storage

$\overline{\mathrm{j}}$ https://www.fujitsu.com/us/Images/Product_Brief_ETERNUS_ DX80-90-S2.pdf. system - as well as its Operating System — is entirely dedicated for this, we have the maximum flexibility and efficiency during intensive observations. Obviously, as well known, RAID 0 is entirely unsafe for the data in case of malfunctioning, however, we do not eliminate the data from the nodes until they are retrieved and archived.

Finally, in order to work properly, the ADCs need a clock signal, so we built a clock distributor. A Valon $5009^{(\mathrm{k})}$ synthesizer is used as a centerpiece, it can provide a clock frequency in the $0.25-6 \mathrm{GHz}$ frequency range, with an output power from $-15 \mathrm{dBm}$ to $+15 \mathrm{dBm}$. Since we need to get 14 clock signals in order to feed the 14 ADCs, we employed an amplifier Mini Circuits ZFL-2500VH+ ${ }^{(1)}$ followed by a 16-way splitter Mini Circuits ZC16PD$252-\mathrm{S}+,{ }^{(\mathrm{m})}$ both with a frequency range from $0.01 \mathrm{GHz}$ to $2.5 \mathrm{GHz}$. A $100 \mathrm{MHz}$ external reference is provided by a H-Maser available at SRT and injected into the synthesizer.

In addition, ADCs need a PPS signal. These signals allow the data capture on the same rising edge of the PPS; this guarantees a proper synchronization

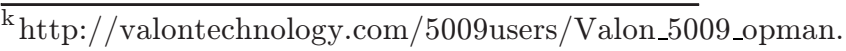
pdf.

${ }^{1}$ https://ww2.minicircuits.com/pdfs/ZFL-2500VH.pdf.

${ }^{\mathrm{m}}$ https://ww2.minicircuits.com/pdfs/ZC16PD-252+.pdf.
} 
between ADCs of the same ROACH2 and, bearing in mind the unavoidable cable propagation differences, between all the ROACH2s. In this case, we used a Timetech Pulse Distribution Unit, ${ }^{(n)}$ which provides PPS pulses to 16 outputs simultaneously; the PPS reference in input is derived from the H-Maser.

\section{Interfacing SARDARA with the Telescope Control Software}

The development of a new digital backend usually requires a large effort. In particular, once assembled and tested in the laboratory, the backend has to be interfaced with the telescope system and validated with observations of test astronomical sources; the way the control software operates the telescope and backends has a large impact on this process. In order to speed up the process, piggy-back observations are often used, as is the case with projects that get little dedicated time, such as the Search for ExtraTerrestrial Intelligence (Lampton et al., 1992). In most other cases, however, the trailing backend is driven by the antenna control system and collects all the ancillary data itself.

In the case of the SRT, the control software can only drive one backend at a time (Orlati et al., 2012), making it virtually impossible to conduct observations in piggy-back mode (with the backend under development) in the conventional way

\footnotetext{
${ }^{\mathrm{n}}$ http://www.timetech.de/10188_Datasheet.pdf.
}

(in parallel with scheduled observations conducted with a leading backend).

To overcome this limitation, we use an approach where the trailing backend is not integrated within the control system, but only acquires and processes sky data, while relying on the files generated by the leading backend for all the ancillary data. The leading backend is fully integrated within the telescope system and is able to collect the appropriately synchronized support data. Therefore, after an observation, the dataset collected by the backend under development will consist of two types of files: the file generated by the backend itself with only the sky data, and a file generated by the leading backend also containing all the required ancillary data. These two files are then merged to generate a final, self-consistent file with appropriately-synchronized sky and ancillary data. Figure 3 shows this scenario graphically. As described in Scalambra et al. (2013), the infrastructure containing the TP also includes the selection of the IFs coming from the three telescope's foci; this infrastructure, named "Focus Selector (FS)", also provides three copies (one dedicated to feed the TP) of the selected signal, thus we exploit one of them in order to feed the two backends simultaneously.

In our specific case, the SARDARA backend is piggy-backing on the TP backend, which has the benefit of offering a fast dump time (down to $1 \mathrm{~ms}$ ) and generating very small data files; this allows us to avoid a high-speed connection between the

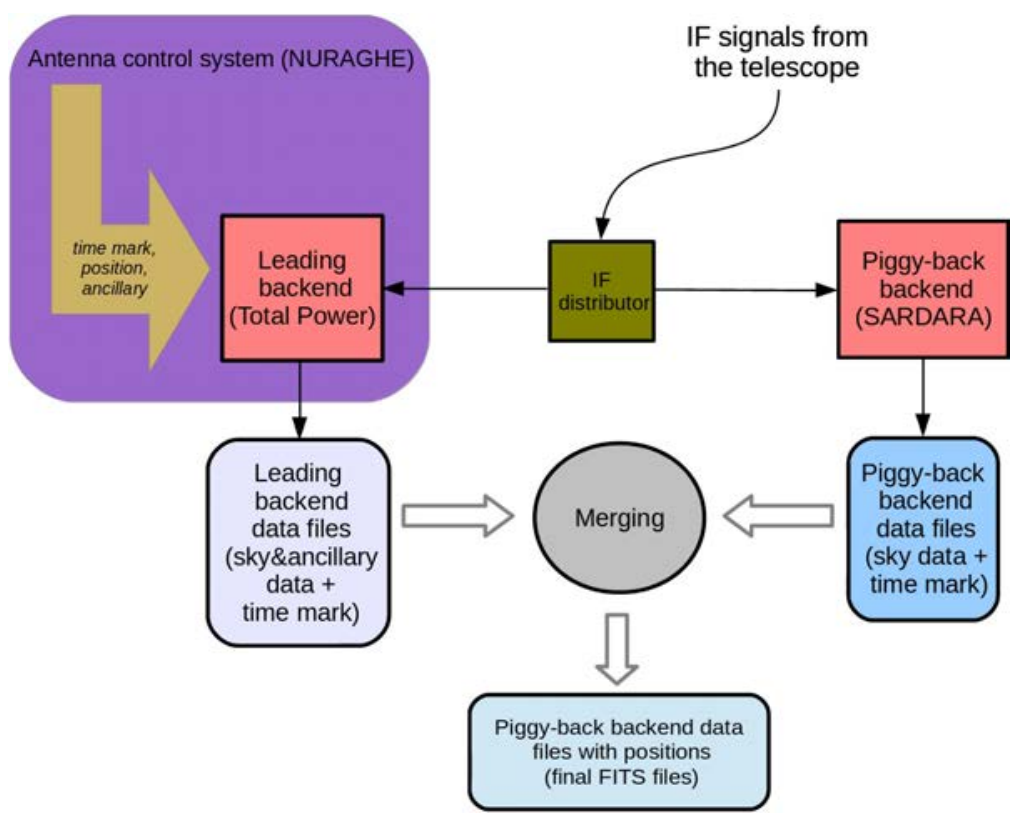

Fig. 3. Block diagram of the backend development technique described in the text. 
computer where the TP data are written and the one where the merging is performed.

\subsection{Description of the technique}

The method we employed can be summarized as follows:

- Observations are conducted with the leading backend (TP in our case), while the backend under test (SARDARA in our case) is piggybacking on the latter and is disconnected from the telescope software control system. The control system generates time marks that are included in the leading backend files containing the ancillary data.

- The trailing backend (SARDARA) works in stand-alone mode, asynchronously recording the sky data only, in the format that is required by its configuration and with a time mark for each time sample (for instance for each integrated spectrum) generated by the backend.

- The time marks are essential for synchronizing the (sky and ancillary) data taken by the two backends and assigning a position to the sky data collected by the trailing instrument.

- The output files of the leading backend provide the ancillary information that is then associated with the sky data taken by the trailing backend by applying a linear interpolation between corresponding times.

- Such a synchronization requires that the two backends use the same reference time. This is ensured by locking both backends to the station's PPS signal, which is driven by a H-maser clock and which aligns all station equipments to a precision of two parts in $10^{-16}$ on long time scales (1000 s).

- Finally, the output sky data taken by the trailing backend are merged offline with the ancillary information taken by the leading backend, thus generating an output file that contains all the required information for subsequent data processing.

In order to implement this method, we used conventional observations with the TP backend, such as those performed during approved science projects. We focused in particular on OTF mapping observations with fast scans and a high dump rate, which enable us to perform our synchronization with a very high time precision. This approach constitutes an excellent test bench for testing whether the linear interpolation is sufficient, given the sampling periods of the data and metadata.
Only at a second stage, the developer will focus on the full integration into the telescope system, by managing the direct acquisition of the ancillary data and making the backend independent and fully integrated.

The high-speed data coming from the ROACH2 boards and handled by the dedicated control computers are first written in an incomplete FITS (Flexible Image Transport System) format file that only contains sky data and the corresponding timemarks. Although there is no specific need to use the FITS format (the data could have been saved in an unformatted binary), we preferred this option to make it possible to use standard astronomical data visualization software for inspecting the file content (e.g. FITSViewer ${ }^{(\mathrm{o})}$ ).

The time mark assigned to each piece of data is the key information contained in the files since it is the sole way to cross-match the two datasets and synchronize sky data with the ancillary data carried by the leading backend files.

\subsection{Merging complete TP FITS files with "data-only" FITS files}

The FITS files produced by the leading and trailing backends are merged to obtain the final and complete FITS files of the (trailing) backend under development. A key feature of this technique is that, since the two systems are entirely independent, the merging can be done either offline or in near real-time. Here, we describe the former: the merging is done offline using a process that runs in parallel with the observations. This process continuously checks for the presence of new files and makes new merged files; seconds later, the temporary files are written into disk. The FITS files produced by the TP are uploaded to the computer that executes the merging soon after they are written. These files are small (tens of $\mathrm{kB}$ ) and a conventional ethernet connection between the two systems (the computer controlling the telescope and the computer controlling SARDARA) is sufficient. However, if for any reason the connection drops, the failing of the real-time merging is not a major issue. The observations can keep going and the files are temporarily recorded, while the merging can be run at a later time.

Figure 4 shows our first successful experiment: $7 \mathrm{GHz}$ observations of the Supernova Remnant (SNR) W44, a diffuse and bright radio astronomical source. The maps were obtained with the TP

\footnotetext{
${ }^{\circ}$ http://heasarc.gsfc.nasa.gov/ftools/fv/.
} 

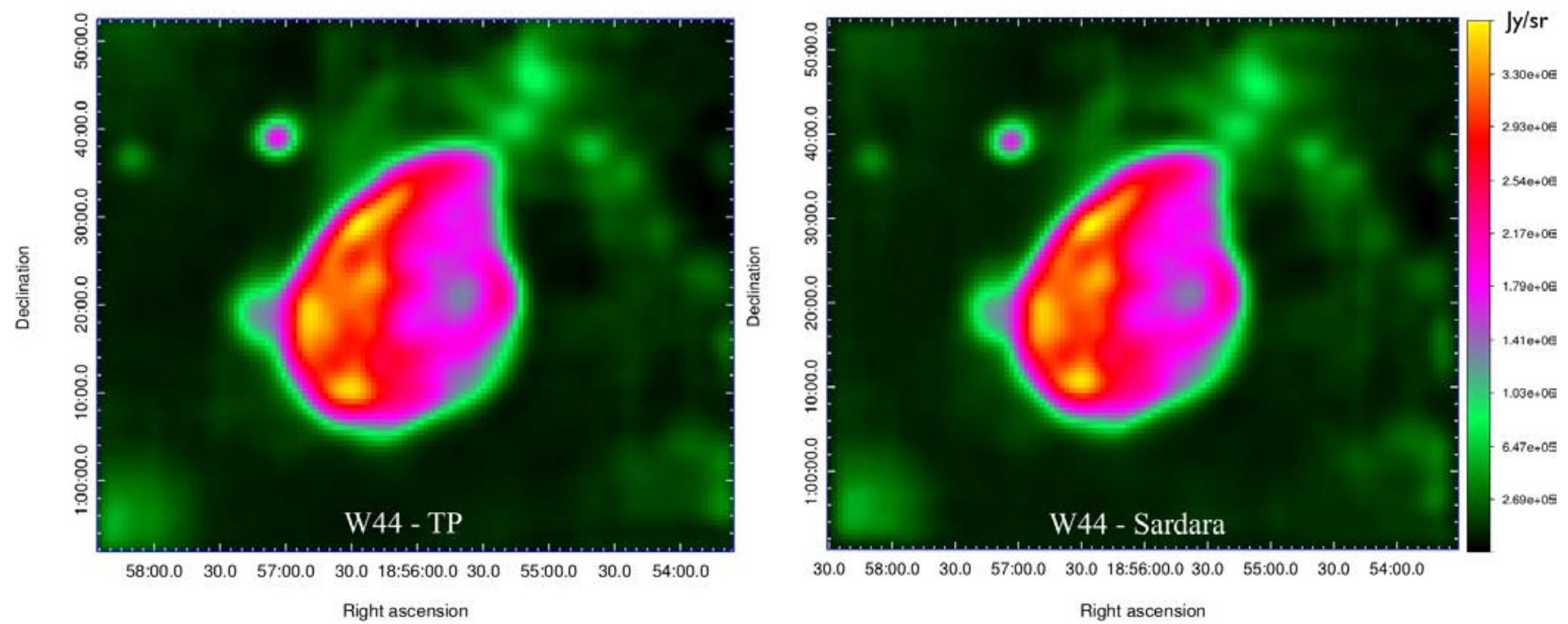

Fig. 4. SNR W44 at $7.0 \mathrm{GHz}$ observed simultaneously with the TP (left panel) and SARDARA (right panel) backends. The used configuration had a bandwidth of $1250 \mathrm{MHz}, 1024$ frequency channels, and $10 \mathrm{~ms}$ of dump time (100 spectra/s). Observation details are as follows: scan speed of $4^{\circ} / \mathrm{min}$, beam size of $2.7 \mathrm{arcmin}$, pixel size of $0.6 \mathrm{arcmin}$, and RMS sensitivity of $7 \mathrm{mJy} /$ beam (SARDARA) and $11 \mathrm{mJy} /$ beam (TP).

leading (left panel) and SARDARA in piggy-back mode (right panel). The comparison of the maps shows the excellent match of the astrometry, thus demonstrating that this technique is successful.

It is worth noting that the map obtained with SARDARA is of better quality: its RMS sensitivity is $7 \mathrm{mJy} /$ beam, which is better than the $11 \mathrm{mJy} /$ beam obtained with the TP data. This is because SARDARA can flag out frequency channels that are contaminated by Radio Frequency Interference (RFI), which the TP cannot do and so retains the additional noise caused by this RFI.

\subsection{Merging algorithm}

A key point of our merging algorithm is the interpolation of the ancillary (sky position) data to the time of the sky data taken by the trailing backend.

Each spectrum delivered by the backend has a time mark assigned by the backend control computer. Let $T s^{r}$ denote the acquisition time of the $r$ th piece of sky data detected (where $r \in[1, \ldots, N]$ and $N$ is the number of spectra), and $T a^{p}$ is the time of the $p$ th piece of ancillary data collected by the leading backend from the antenna telescope control software (where $p \in[1, \ldots, M]$ and $M$ is the number of ancillary data samples). It is not a requirement to take sky and ancillary data taken with the same sampling interval, and $N$ and $M$ are usually different.

The first step is to associate each time $T s^{r}$ with the nearest value of $T a^{p}$. This is done with a standard binary search algorithm (Oommen et al., 2014); the telescope coordinate (i.e. either right ascension (RA), declination (DEC), azimuth, or elevation) - that is associated with the piece of sky data - is hence obtained by linear interpolation.

Once these coordinates are calculated, a final and complete FITS file is written by adding these values to the sky data from the temporary sky-dataonly FITS file. This method thus delivers FITS files that are complete with reliable ancillary (sky position) information.

With this approach, development observations can be done in piggy-back mode with previously scheduled astronomical observations (a possibility that is not built-in in the SRT control system), and the astronomer conducting the leading observations can provide support and give quick feedback on the data taken with the new instrumentation. In this way, the amount of telescope time solely dedicated to development is reduced to that needed for the final testing before the new instruments are officially offered to the community. On the other hand, astronomers who provide support gain early access to the new instrument, and benefit in advance of its better performance and new observing modes, which are not yet offered to the community.

\section{Scientific Observational Results}

In this section, we present some examples of scientific observations that were conducted at the SRT with 
the SARDARA backend in different observing modes: imaging, polarimetry, spectroscopy, and pulsars at high time resolution. All the observations were conducted with TP schedules and FITS files obtained with the merging method described in Sec. 3.

\subsection{Imaging}

The radio continuum mapping of astrophysical sources using single-dish observations can compete with interferometric imaging when coupled with innovative observing modes with a flexible backend such as SARDARA. One of the challenging scientific cases for the SRT is the study of bright Galactic extended sources (such as SNRs) using OTF mapping techniques (Egron et al., 2017). SNRs are a manifestation of the final episode in the evolution of a massive star, namely the explosion of the star in a supernova event.

Most studied SNRs are typically bright (with a flux density of about 10-100 Jy at $1 \mathrm{GHz}$ ), extended sources $\left(0.5-1^{\circ}\right)$ that were well suited to perform the "first light" of SARDARA. Integrated SNR fluxes are typically available in the literature for up to 5-10 GHz, while spatially-resolved fluxes are largely unexplored above this frequency range. W44 is one of the best-studied Galactic SNRs. The large structure of the source extends over $0.75^{\circ}$ and shows evidence of interactions with both atomic and molecular clouds (Yoshiike et al., 2013). SARDARA obtained the first high-resolution images of SNR W44 at $7 \mathrm{GHz}$, with a better sensitivity with respect to the simultaneous piggy-back TP observations (Fig. 4). For these observations, the OTF data acquisition was performed with continuity (with a dump time of a few tens of ms) at a constant antenna speed (typically a few degrees/min), repeatedly scanning the sky in both RA and DEC (each passage is called a "subscan", while the full collection of subscans is called a "scan") around the centroid of the source. The data reduction was based on a state-ofthe-art imaging data analysis software optimized for OTF scans with SARDARA, which includes automated baseline subtraction techniques and RFI rejection (Egron et al., 2017). L-band observations (Valente et al., 2010) at the SRT site are greatly affected by RFI. For this reason, radio continuum imaging with the TP backend is worthless, since the data is unavoidably contaminated by RFI that prevents a proper calibration. Instead, the use of the SARDARA backend allows us to dynamically and

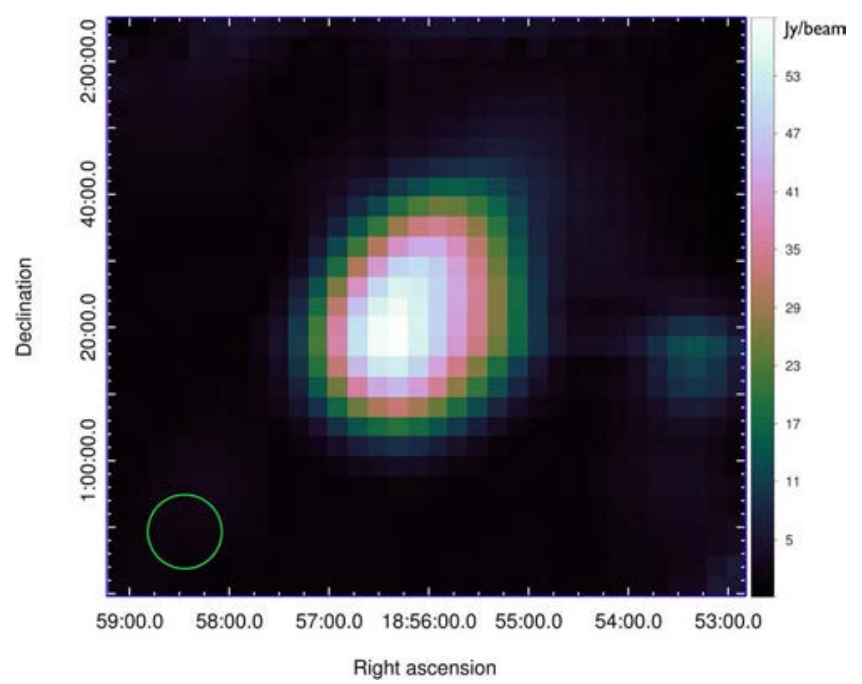

Fig. 5. OTF map of SNR W44 performed with SARDARA at $1.5 \mathrm{GHz}$. The beam size of 11.1 arcmin is indicated on the bottom left of the map by a circle. The adopted configuration has a bandwidth of $1500 \mathrm{MHz}$ divided into 1024 spectral channels for both polarizations, scan speed of $4 / \mathrm{min}$, pixel size of 3 arcmin, and RMS sensitivity of $81 \mathrm{mJy} /$ beam.

selectively remove the spectral channels that are affected by RFI. Figure 5 shows a typical example of this observing mode, performed on 2016 February 23 for which the RFI situation was bad. The spectra rate was 50 spectra/s.

The radio continuum map, obtained with SARDARA at $1.5 \mathrm{GHz}$ with a $3^{\prime}$ resolution, appeared to be totally free from RFI contamination after proper spectral filtering. The flux density associated with SNR W44 is $214 \pm 6 \mathrm{Jy}$, which is consistent with previous measurements obtained at $1.4 \mathrm{GHz}(210 \pm 20 \mathrm{Jy})$ (Giacani et al., 1997). Each map's pixel represents the overall received energy for the corresponding sky area. The adopted configuration provides a bandwidth of $1500 \mathrm{MHz}$ divided into 1024 spectral channels for both polarizations. After RFI removal (about 30\% of spectral channels were affected), all of the channels are "collapsed" into a single one in order to obtain the power over the entire bandwidth.

\subsection{Polarimetry}

In Fig. 6, we show total intensity and polarization observations of the Virgo A radio source, obtained with the SRT using the central feed of the K-band receiver.

Virgo A is the fourth brightest radio source in the northern sky (with a flux density of more than $200 \mathrm{Jy}$ at $1.4 \mathrm{GHz}$ ). The tool adopted to calibrate 

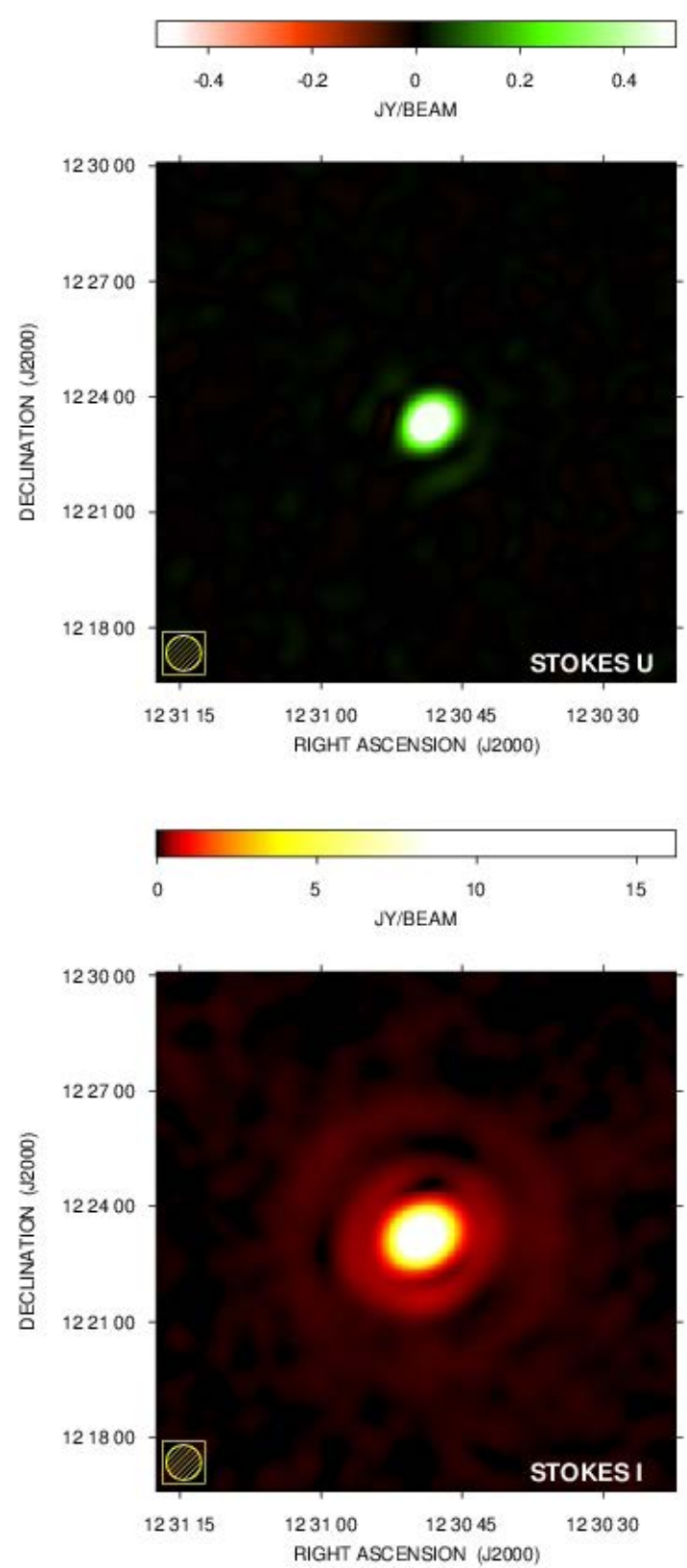
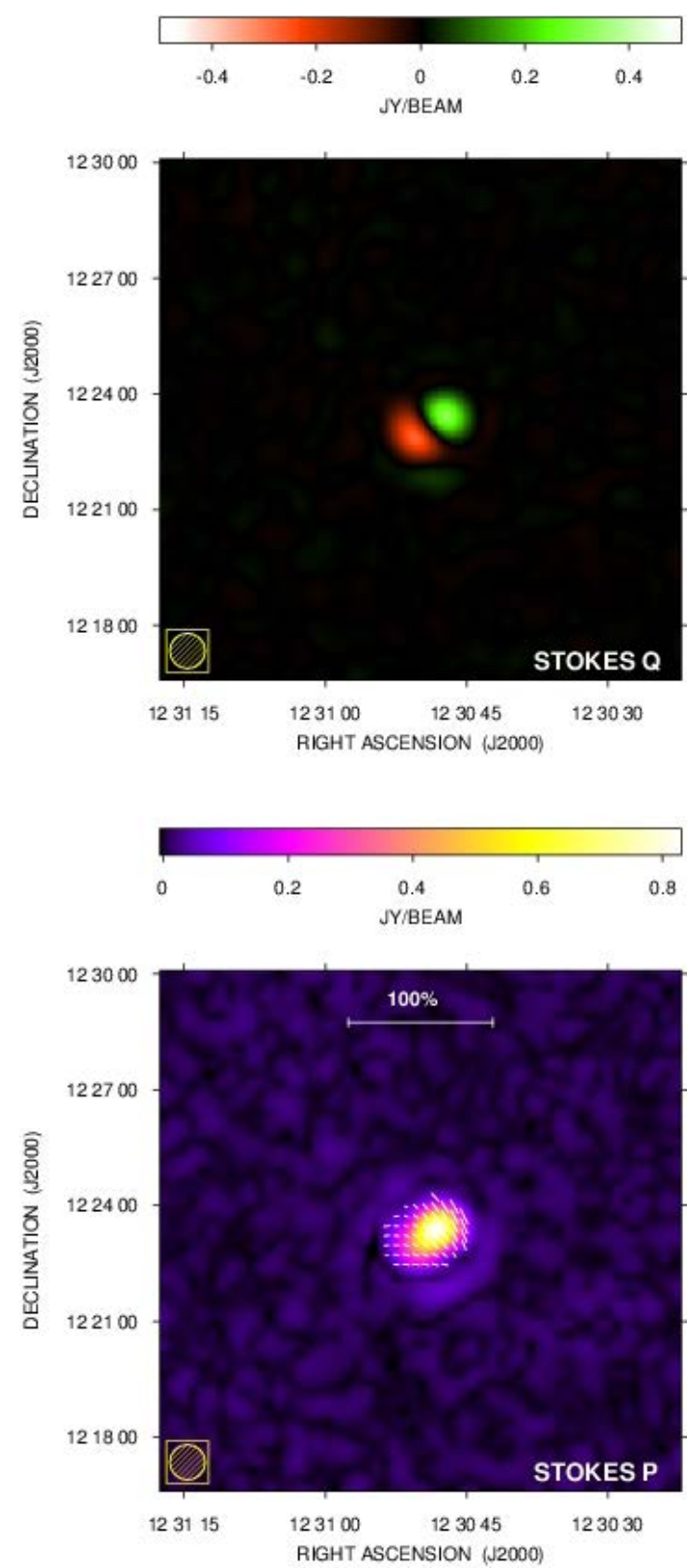

Fig. 6. SRT total intensity and polarization images of Virgo A with SARDARA resulting from the spectral average of the bandwidth between $18 \mathrm{GHz}$ and $20 \mathrm{GHz}$, see text. The top-left and top-right panels show the images of Stokes parameters U and Q, respectively. The bottom-left panel shows the total intensity image. The bottom-right panel shows the polarization intensity $P=\sqrt{U^{2}+Q^{2}}$ with the polarization vector over imposed. The length of the vectors is proportional to the fraction polarization while the polarization position angle represents the direction of the E-field (not corrected for the Faraday rotation effect).

and image all of the observations is named Single-dish Spectral-polarimetry Software (SCUBE) (Murgia et al., 2016); it has been applied during the SRT's early science program in order to study the polarization properties of radio sources in galaxy clusters (Govoni et al., 2017; Loi et al., 2017).

This test observation was acquired on 2015 November 15. It consists of the average of five scans along RA and five scans along the DEC direction.
The telescope scanning speed was $3 \mathrm{arcmin} / \mathrm{s}$ and full-Stokes spectra were acquired every 4 milliseconds (244 spectra/s), with a bandwidth of $2 \mathrm{GHz}$ and 1024 channels. The bandwidth is centered at a frequency of $19 \mathrm{GHz}$. Data calibration and imaging were performed by using the calibrator $3 \mathrm{C} 286$ for flux density and for the absolute position of the polarization angle. The on-axis instrumental polarization was determined and corrected using the 
radio source $3 \mathrm{C} 84$, which we assumed to be unpolarized. The imaging of Stokes parameters Q, U and I, is performed in SCUBE by subtracting the baseline from the calibrated telescope scans and by projecting the data into a regular three-dimensional grid with a resolution of 15 / / pixel, which is enough in our case for sampling the beam Full Width at Half Maximum (FWHM) of 55". The spectral cubes along the RA and DEC directions were stacked together by mixing their Stationary Wavelet Transform (SWT) decompositions (see Murgia et al. (2016)) in order to remove residual scanning noise and to increase the signal-tonoise $(\mathrm{S} / \mathrm{N})$ ratio.

To further increase the image sensitivity, we averaged together all of the 1024 channels for both the total intensity and the $\mathrm{Q}$ and $\mathrm{U}$ cubes. The final RMS noise level in the total intensity image is about $6 \mathrm{mJy} /$ beam, while the final RMS noise level in the images of $\mathrm{Q}$ and $\mathrm{U}$ parameters is $4 \mathrm{mJy} /$ beam. We clearly resolved the bright inner double radio source that hosts the core of the radio galaxy and the famous one-sided radio jets.

In the bottom-right panel of Fig. 6, we also show the polarized intensity $\left(P=\sqrt{U^{2}+Q^{2}}\right)$, which was corrected for the bias introduced when combining the Stokes Q and U images and that we assumed to have an equal noise Gaussian statistics with a measured standard deviation $\sigma_{Q, U}=4 \mathrm{mJy} /$ beam. The peak polarized intensity is $0.83 \mathrm{Jy} /$ beam, which is about $5 \%$ of the total intensity peak of $16.2 \mathrm{Jy} /$ beam. The average polarization percentage is $6 \%$. The SRT image shows that the polarization vectors rotate from the west side of the inner double (where they have a position angle of $-45^{\circ}$ ) to the east side, where they are almost horizontal (position angle of $-90^{\circ}$ ).

A polarization image of Virgo $\mathrm{A}$ - obtained with the Effelsberg $100-\mathrm{m}$ radio single-dish at $10.6 \mathrm{GHz}-$ was presented by Rottmann et al. (1996), whose images show a larger field of view, due to the better sensitivity. However, at least for the central part of the source, one can compare the B-field polarization vectors (see Fig. 1 of Rottmann et al. (1996)) with the E-field vectors in Fig. 6 by taking into account the fact that they are rotated by $90^{\circ}$. Overall, the polarization images match although, given the different frequencies of the two observations, some differences may be present due to the Faraday rotation effect.

\subsection{Spectroscopy}

The scientific detailed analysis of emission/absorption spectral lines (i.e. line profiles, intensity line ratios, etc.) requires a high-frequency resolution that is well above the one employed for the studies outlined in previous sections (e.g. the $1.4 \mathrm{MHz}$ channel spacing used for spectropolarimetric imaging). In this case, a configuration with 16,384 channels was used, with different bandwidths for two different targets. Figure 7 shows the first observation, i.e. the spectrum of the $22-\mathrm{GHz}$ water maser source observed in NGC4258 (Bragg et al., 2000), which was taken with the SRT using SARDARA on 2015 October 24.

The total bandwidth is $1.5 \mathrm{GHz}$ and was split into 16,384 channels, yielding a full velocity coverage (only a portion of it is shown in the figure) and a channel spacing of 20,450 and $1.2 \mathrm{~km} / \mathrm{s}$, respectively. The frame of the velocity reported in the $x$-axis is heliocentric.

The spectrum shows the redshifted high-velocity features (red, in a velocity range of 1200-1350 km/s), the weaker blueshifted high-velocity features (blue, around $-500 \mathrm{~km} / \mathrm{s}$ ), and the systemic velocity features (from $400 \mathrm{~km} / \mathrm{s}$ to $600 \mathrm{~km} / \mathrm{s}$ ). The systemic velocity of the galaxy is $448 \mathrm{~km} / \mathrm{s}$.

The spectrum is consistent - within the uncertainties and accounting for the variability of the emission - in the flux densities and velocity ranges of the line groups with those taken at other single-dish facilities at many epochs (see e.g. Bragg et al. (2000) and references therein). The total integration time of the SRT measurement was of about $40 \mathrm{~min}$, yielding a spectrum RMS noise level of $\sim 10 \mathrm{mJy} /$ beam $/$ chan. This result shows the capability of the SRT to detect, in a reasonable amount of time (comparable to that necessary by a 100-m class radio telescope without active optics), relatively weak features, like the (weakest) blueshifted ones in NGC4258 shown in Fig. 8 (left inset). Indeed, the observations were performed in position switching mode, by using only one (the central) of the seven feeds of the SRT K-band receiver, thus spending half of the observing time off source. The performances of the SRT in detecting/ monitoring spectral line at K-band (e.g. water masers) will thus be further enhanced when observations in "nodding mode"(p) are available. Recent spectroscopic tests using the nodding mode have

\footnotetext{
p The nodding technique is a version of position switching for receivers with multiple beams, e.g. two beams $\mathrm{A}$ and $\mathrm{B}$. When A is on source, B is off source (on blank sky). Then the telescope is moved so that B is on source and A is on blank sky for an equal time. This allows position switching with no lost time while performing the off-source observations.
} 


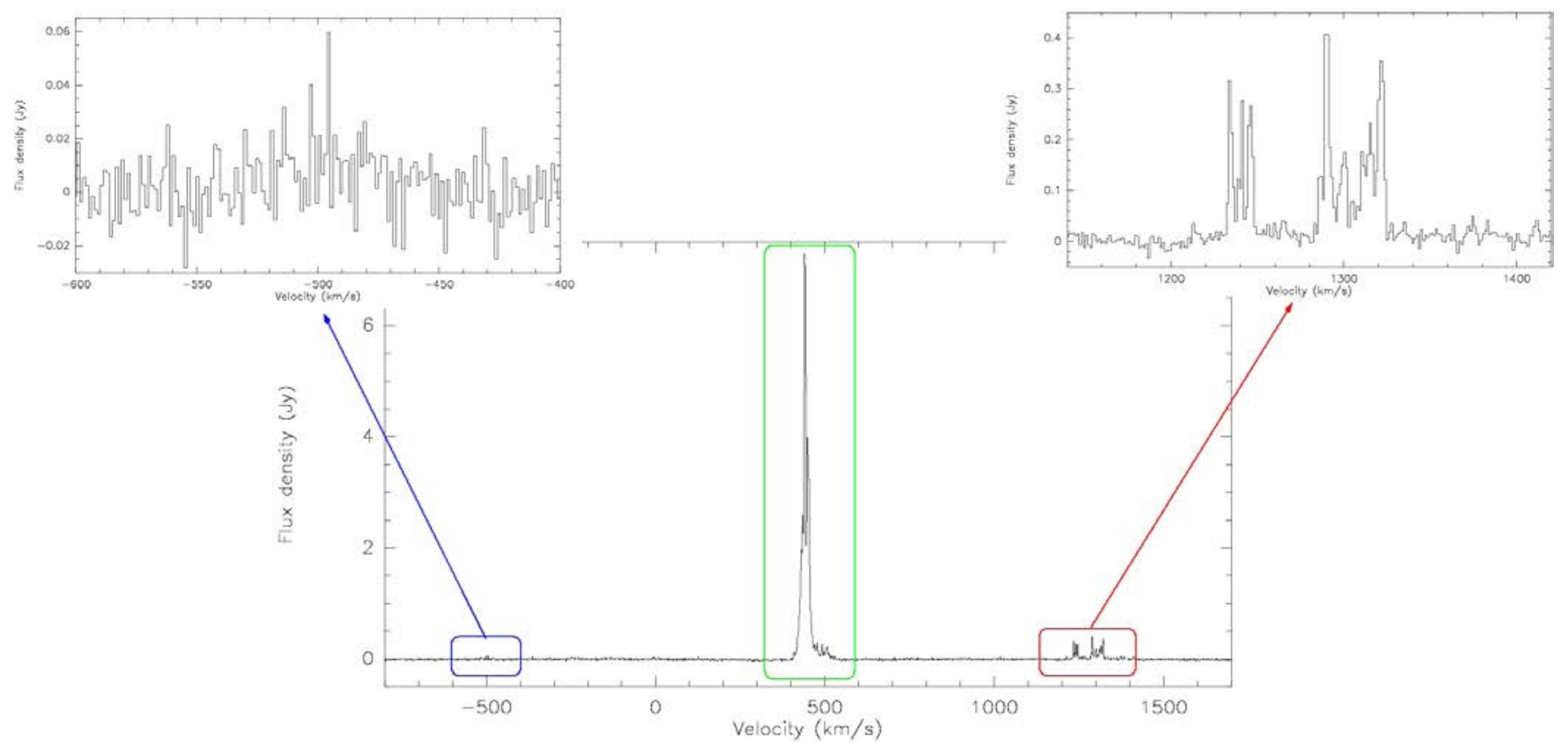

Fig. 7. $\mathrm{H}_{2} \mathrm{O}$ maser spectrum observed towards the galaxy NGC 4258 with the SRT at K-band using SARDARA. The backend was configured with a bandwidth of $1.5 \mathrm{GHz}$ and 16,384 channels, yielding a channel spacing of $91 \mathrm{kHz}$, corresponding to $1.2 \mathrm{~km} \mathrm{~s}^{-1}$ at $22 \mathrm{GHz}$. The source was observed in position-switching mode for a total observing time of about $40 \mathrm{~min}$ (20 min on-source). The final RMS of the spectrum is of $\sim 10 \mathrm{mJy} /$ beam/chan. The green box indicates the features close to the systemic velocity of the galaxy $\left(448 \mathrm{~km} \mathrm{~s}^{-1}\right)$. The blue and red boxes, instead, mark the blueshifted and redshifted high-velocity components, respectively. A zoom of these features is shown in the corresponding insets. The velocity scale is with respect to the heliocentric frame and uses the optical convention.

been successfully led with the SRT at K-band, showing promising results (Tarchi et al., 2018). The three groups of lines represent distinctive characteristics of masers associated with accretion disks orbiting supermassive black holes in Active Galactic Nuclei (AGN). The combination of single-dish monitoring and high-angular resolution maps (in particular taken with Very Long Baseline Interferometry - VLBI - arrays) of the maser lines allows us to shape the rotation curve of the accretion disk, derives the distance to the host galaxy, and estimates the black hole mass, as is now successfully done for a number of galaxies like NGC4258 (Bragg et al., 2000) and UGC3789 (Braatz et al., 2010).

Spectroscopic images (typically referred to as "cubes") can also be produced using SARDARA. M31 (Spencer et al., 2013), also known as the Andromeda Galaxy, is a spiral galaxy that is approximately 780 kiloparsecs from Earth. Andromeda is the largest galaxy (roughly 220,000 light years across) of the Local Group (the latter also contains the Milky Way, the Triangulum Galaxy as well as about 44 other smaller galaxies). The interstellar medium in M31 contains about $7.2 \times 10^{9}$ solar masses of neutral hydrogen (HI), thus a spectral line at a rest frequency of $1420.4 \mathrm{MHz}$ is produced. We observed M31 on 2015 September 16 with SRT; the aim was to test the spectral imaging capabilities of SARDARA. Two OTF maps of a field-of-view of about $5 \times 5$ square degrees were obtained along the RA and DEC directions. We acquired a bandwidth of $300 \mathrm{MHz}$, starting at $1250 \mathrm{MHz}$ and with 16,384 spectral channels of $18.3 \mathrm{kHz}$ in width. Data reduction was performed with the SCUBE software package; RFI was excised using an automated procedure, we corrected for bandpass and removed the scan baseline. The raw counts were calibrated to Jansky/beam using the well-known calibrator 3C286. Finally, we combined two scans along RA and DEC in a single spectral cube. A continuum subtracted moment- 0 image of the $\mathrm{HI}$ emission is presented in Fig. 8. Colors indicate the Doppler shift of the HI line, caused by the rotation of the M31 galaxy disk. The North East tip of the disk has a receding radial velocity of about $200 \mathrm{~km} / \mathrm{s}$, with respect to the systemic velocity and, therefore, appears redshifted while the South West tip is blueshifted by the same amount. 

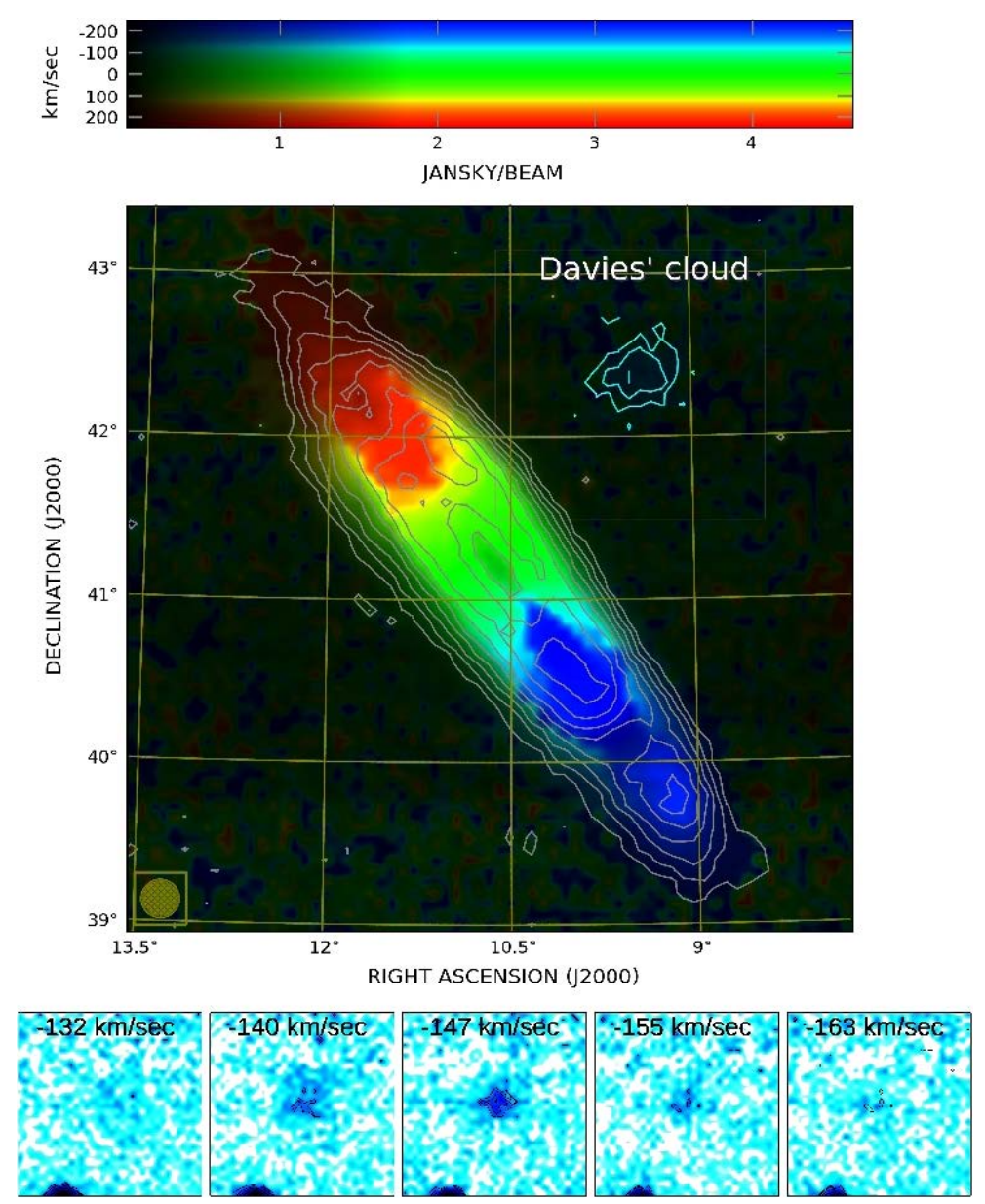

Fig. 8. Andromeda galaxy (M31) observed at SRT with SARDARA. Details in the text. Contour levels refer to the continuumsubtracted HI image, they start at $0.3 \mathrm{Jy} /$ beam, 14 arcmin beam, and increase by $\sqrt{2}$. Colors refer to the radial velocity of the HI gas after the subtraction of the systematic velocity of M31, which is approaching our solar system with a radial velocity of $-300 \mathrm{~km} / \mathrm{sec}$. Red tones correspond to receding velocities while blue tones represent the approaching gas. The color refers to the peak of the HI line profile, while the intensity of each pixel represents the average over the bandwidth. The contours in the top panel represent the average intensity of the Davies's cloud integrated in the velocity range from $-128 \mathrm{~km} / \mathrm{s}$ to $-167 \mathrm{~km} / \mathrm{s}$. Bottom panels show the cloud emission in a few selected velocity channels.

An interesting HI feature detected in the SRT image is the isolated HI cloud located north west of the center of M31. The cloud was discovered by Davies et al. (1975) and studied in detail by Heij et al. (2002). The contours in the top panel represent the average intensity of the Davies's cloud integrated in the velocity ranging from $-128 \mathrm{~km} / \mathrm{s}$ to $-167 \mathrm{~km} / \mathrm{s}$. Bottom panels show the cloud emission in a few selected velocity channels.

\subsection{High time resolution observations}

In the following, we present two test pulsars observed in total intensity. Figure 9(a) shows a 3-min long observation of PSR B0355+54 (Manchester et al., 1972), which is a pulsar with a period of roughly $0.15 \mathrm{~s}$ and a DM of $57.1 \mathrm{pc} / \mathrm{cm}^{3}$.
The observation was performed on 2015 December 15 with the C-band receiver (Navarrini et al., 2017) with a central frequency of $6400 \mathrm{MHz}$ and a 500-MHz bandwidth divided into $3421.4 \mathrm{MHz}$-wide frequency channels. The plot shows the pulsar profile in arbitrary flux units and the profile phase evolution with frequency. Given the specificity of pulsar observations, in order to check the data quality, we performed a simultaneous acquisition of the same pulsar using the DFB3 backend, which uses a dedicated control software described in Corongiu (2014). The signal was 1-bit sampled every $0.125 \mathrm{~ms}$ and folded offline for the DFB3; $1-\mathrm{MHz}$ channels were used. Figure 9(b) shows the same plot for the corresponding observation. The shape of the pulse profile and the derived pulsar parameters are compatible in the two instruments. 


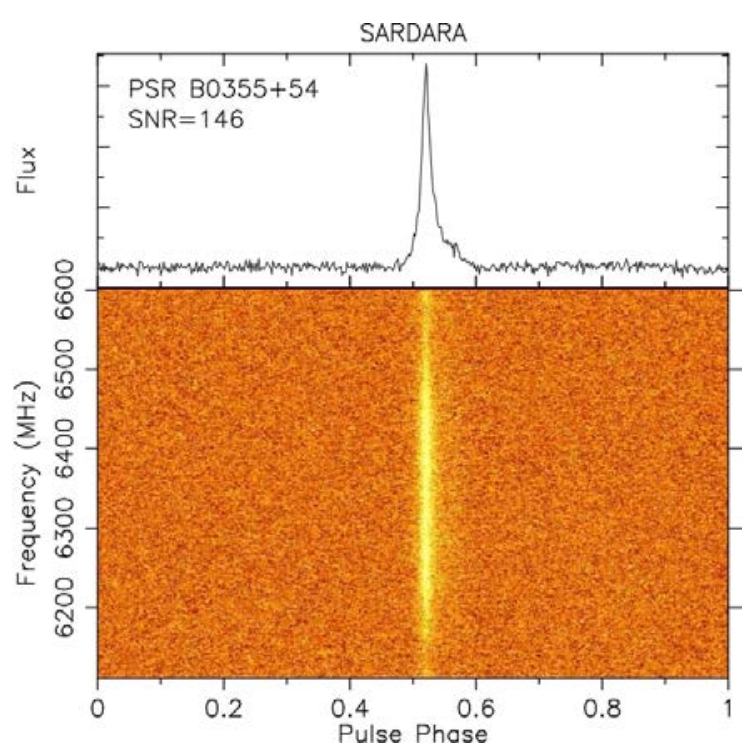

(a)

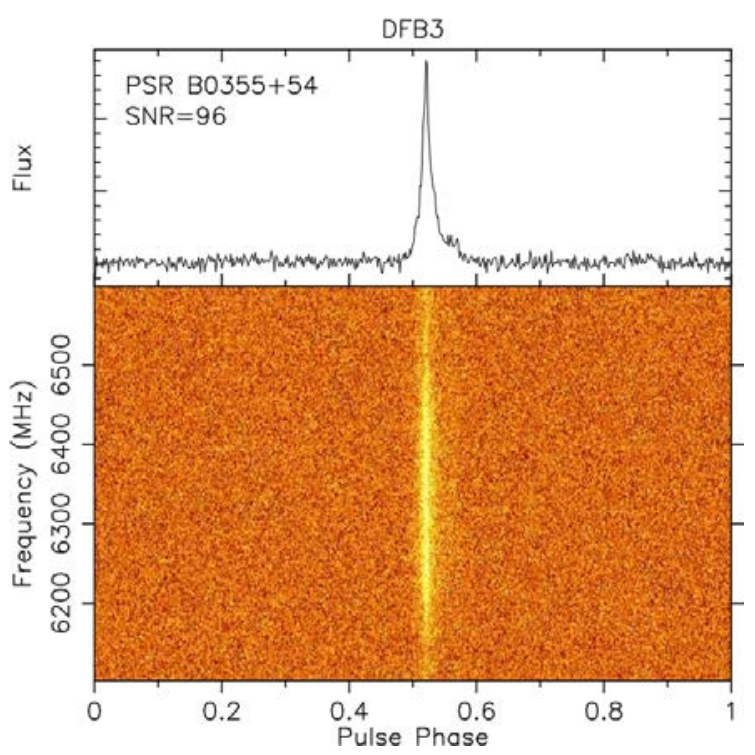

(b)

Fig. 9. Pulsar B0355+54 observed at C-band with the SRT. In the top panel, we show the integrated pulse profile over one rotation in arbitrary flux units; in the bottom panel, we show the frequency dependence of the pulsed emission. Panel (a) represents the 3-min-long observation performed with SARDARA, while panel (b) represents the 3-min-long observation performed simultaneously with the DFB3.

Figure 10(a) shows the folding of a 4-min long observation of PSR B2334+61 (Dewey et al., 1985), a pulsar with a period of roughly $0.49 \mathrm{~s}$ and a DM of $58.4 \mathrm{pc} / \mathrm{cm}^{3}$. The data acquisition was performed on 2016 March 10 with the L-band receiver with a central frequency of $1550 \mathrm{MHz}$ and a $500-\mathrm{MHz}$ bandwidth divided into $3421.4 \mathrm{MHz}$-wide frequency channels. The plot shows the pulsar profile in arbitrary flux units and the profile phase evolution with frequency. For the chosen pulsar, with a relatively

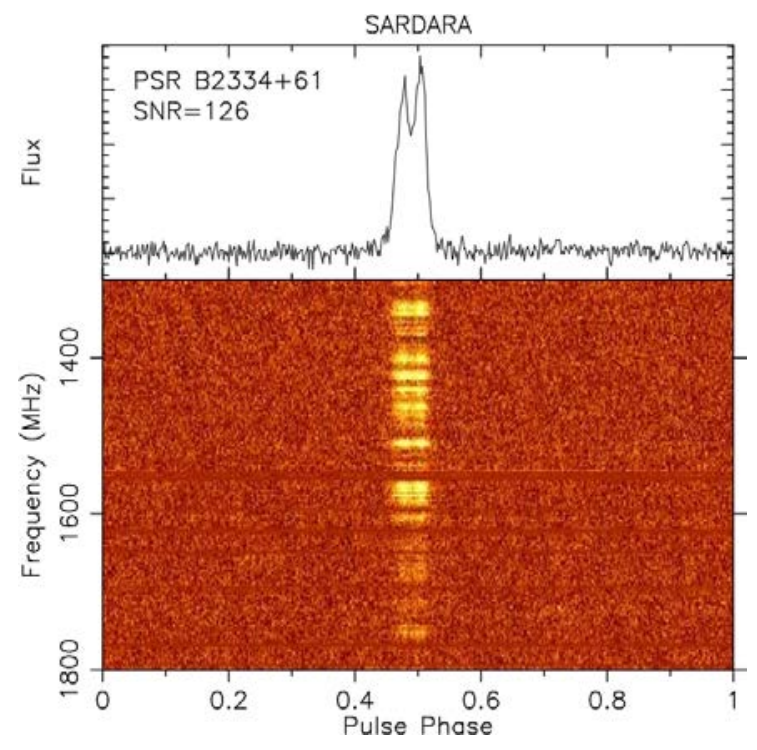

(a)

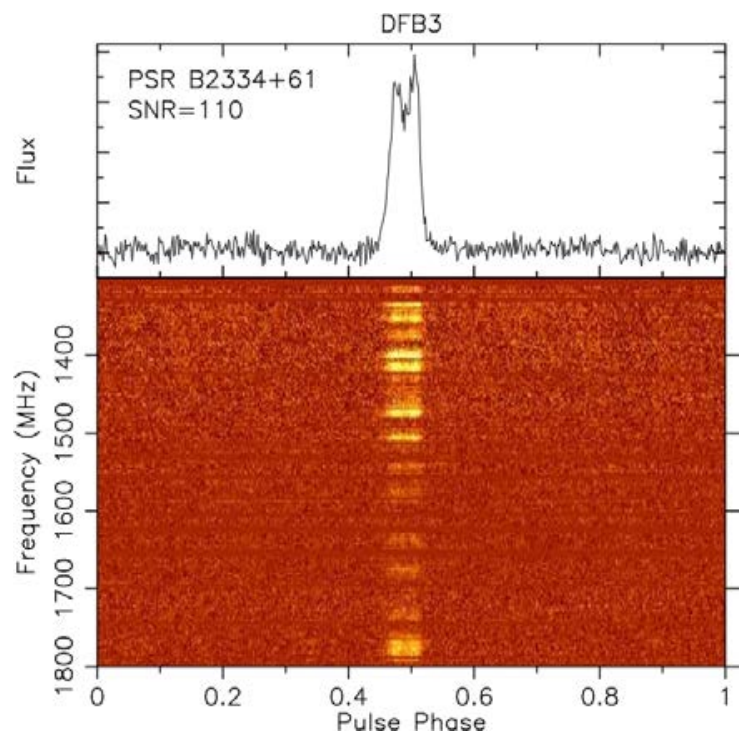

(b)

Fig. 10. Pulsar B2334+61 observed at L-band with the SRT. In the top panel, we show the integrated pulse profile over one rotation in arbitrary flux units; in the bottom panel, we show the frequency dependence of the pulsed emission. Panel (a) represents the 4-min-long observation performed with SARDARA, while panel (b) represents the 4-min-long observation performed on the same day with the DFB3. Both datasets were scrunched in frequency after dedispersion and RFI removal in order to show the same frequency resolution $(3 \mathrm{MHz})$. 
long period and small DM, a particularly high frequency resolution was not required. The corresponding 4-min long observation taken in folding mode with the DFB3 with a central frequency of $1550 \mathrm{MHz}$ and a $512-\mathrm{MHz}$ bandwidth divided into $5121 \mathrm{MHz}$-wide frequency channels is shown in Fig. 10(b). The blank horizontal lines in the phase vs. frequency plot indicate that the corresponding frequency channels were strongly affected by RFI, thus they have been removed. The shape of the pulse profile and the derived pulsar parameters are compatible in the two instruments, taking into account that the observed pulsar is a known scintillator. For both pulsars, the data taken with SARDARA have a dump time of 2000 spectra/s, corresponding to a sampling time of $0.5 \mathrm{~ms}$. The raw data were converted into a standard FITS format suitable for pulsar analysis (psrfits) — and folded offline. The DFB3 observations in both cases show a lower $\mathrm{S} / \mathrm{N}$ ratio at L-band, possibly due to the fact that the DFB3 was more strongly affected by RFI, hence $10 \%$ more band had to be removed. The 1-bit sampling of the DFB3 C-band data (compared to the 8-bit sampling used in SARDARA) has also likely played a role in lowering the $\mathrm{S} / \mathrm{N}$ of the integrated pulse for such a strong pulsar. Finally, we note that the attenuation chain is not the same for the two backends, which could introduce minor differences. A comparison with the literature is not possible for PSR B0355+54, for which the EPN Database $^{(\mathrm{q})}$ includes pulse profiles at $4 \mathrm{GHz}$ and $8 \mathrm{GHz}$, but not at 6 , and for which a $6 \mathrm{GHz}$ flux density measurement has never been published. For $\mathrm{B} 2334+61$, on the other hand, the $20-\mathrm{cm}$ pulse profiles of the EPN Database show the same double peaked structure and the same pulse width that we see in Fig. 10. For this pulsar, we can also make a crude estimate of the flux density through the radiometer equation (Manchester et al., 2001) and compare it with the literature. Considering that, due to a technical malfunction of the noise injector, our measurements are uncalibrated and that the pulsar scintillates the value of $\sim 2 \mathrm{mJy}$ that we obtain using the SARDARA S/N is roughly compatible with the of 1.4(3) mJy obtained by Lorimer et al. (1995).

\section{Conclusions}

We have presented the SARDARA, a new general purpose backend developed for the SRT. This

\footnotetext{
${ }^{\mathrm{q}}$ www.epta.eu.org/epndb/.
}

digital platform was built to overcome limits of the currently available backends, especially in terms of instantaneous bandwidth and processing capability for the seven-feed K-band receiver. We described the commissioning of the backend with single-feed receivers (L-band, C-band, central feed of the $\mathrm{K}$ band) in different observing modes: imaging, polarimetry, spectroscopy, and high-time resolution observations. We also outlined an alternative piggy-back technique used to interface SARDARA with the telescope control software during its development.

Future works mainly regard the multi-feed commissioning of SARDARA. This will be done both to "extend" observing modes described in this paper and to implement new modes: spectroscopy at high-frequency resolution, pulsar timing, and pulsar searching (in particular for millisecond pulsars). For the latter, we will improve both the temporal (more spectra/s) and frequency resolution, and include full-Stokes information. This development is also important in view of a seven-feed S-band receiver (sky frequency range: $3-4.5 \mathrm{GHz}$ ) that is currently under development for the SRT with a high priority.

\section{Acknowledgments}

The development of the SARDARA backend has been funded by the Autonomous Region of Sardinia (RAS) using resources from the Regional Law 7/ 2007 "Promotion of the scientific research and technological innovation in Sardinia" in the context of the research project CRP-49231 (year 2011): "High resolution sampling of the Universe in the radio band: An unprecedented instrument to understand the fundamental laws of nature" P.I. of the project: Dr. Andrea Possenti. F. Loi gratefully acknowledges the Sardinia Regional Government for the financial support of her $\mathrm{PhD}$ scholarship (P.O.R. Sardegna F.S.E. Operational Programme of the Autonomous Region of Sardinia, European Social Fund 2007-2013 - Axis IV Human Resources, Objective 1.3, Line of Activity 1.3.1.). The Sardinia Radio Telescope (SRT, Bolli et al. (2015), Prandoni et al. (2017)) is funded by the Department of University and Research (MIUR), Italian Space Agency (ASI), and the Autonomous Region of Sardinia (RAS) and is operated as National Facility by the National Institute for Astrophysics (INAF). 


\section{Appendix A}

Tables A.1-A.4 show a set of the observing modes that we plan to implement with SARDARA. The various bandwidths were chosen so as to take into account the anti-aliasing filters provided by the FS. The low-pass filters of the FS have nominal cutoff frequencies of $300,730,1250$, and $2100 \mathrm{MHz}$. However, since they are not highly efficient filters and in order to avoid aliasing phenomena, we have to widen the instantaneous bandwidths at the following values: 420, 1000, 1500, and $2200 \mathrm{MHz}$. As for the L-band receiver, an efficient pass-band filter operating in the $500-1000 \mathrm{MHz}$ frequency range is available; this allows us to set an instantaneous bandwidth of $500 \mathrm{MHz}$ (we exploit the second Nyquist window).

Table A.1. WB and WB for Pulsars (WBP) full-Stokes spectrometer modes under development for SARDARA. These modes will be available for 1,2 , or 7 feeds simultaneously.

\begin{tabular}{lcccc}
\hline Mode & BW $(\mathrm{MHz})$ & No. of channels & Spectral resol. $(\mathrm{kHz})$ & Min. Integr. time (ms) \\
\hline WB1 & 420 & 16,384 & 25.6 & 10 \\
WB2 & 1500 & 1024 to 16,384 & 1465 to 91.5 & 10 \\
WB3 & 2200 & 1024 to 16,384 & 2148 to 134.3 & 10 \\
WBP1 & 500 & 1024 to 4096 & 488.2 to 122 & 0.0625 \\
WBP2 & 1500 & 1024 to 4096 & 1465 to 366.2 & 0.0625 \\
\hline
\end{tabular}

Table A.2. WB Pulsar Timing (WBPT) modes under development for SARDARA. The input bandwidth will be split into a number of sub-bands with a parallel PFB; these complex-valued data will then be sent to the GPU cluster and stored or, alternately, post-processed with the standard pulsar pipelines ("dspsr," etc.). These modes will be available for only one feed.

\begin{tabular}{lccc}
\hline Mode & BW $(\mathrm{MHz})$ & No. of sub-bands & Post processing \\
\hline WBPT1 & 500 & 8 to 128 & Baseband recording or folding \\
WBPT2 & 1000 & 8 to 128 & Baseband recording or folding \\
WBPT3 & 1500 & 8 to 128 & Baseband recording or folding \\
\hline
\end{tabular}

Table A.3. WB High Resolution (WBHR) full-Stokes spectrometer modes under development for SARDARA. The input bandwidth will be split into a number of sub-bands with a parallel PFB; these complex-valued data will then be sent to the GPU cluster, and a second PFB will be applied for each sub-band; finally, all of the spectra will be stitched together in order to compose the final spectrum. These modes will be available for only one feed.

\begin{tabular}{lcccc}
\hline Mode & BW $(\mathrm{MHz})$ & No. of channels & Spectral resol. (kHz) & Min. Integr. time (ms) \\
\hline WBHR1 & 1000 & 32,768 to $4,194,304$ & 30.5 to 0.238 & 10 \\
WBHR2 & 1500 & 32,768 to $2,097,152$ & 45.7 to 0.715 & 10 \\
\hline
\end{tabular}


Table A.4. NB and Zoom (Z) full-Stokes spectrometer modes under development for SARDARA. These modes will be available for 1, 2, or 7 feeds.

\begin{tabular}{lcccc}
\hline Mode & BW $(\mathrm{MHz})$ & No. of channels & Spectral resol. (kHz) & Min. Integr. time (ms) \\
\hline NB & 150 & 16,384 to 524,288 & 9.1 to 0.286 & 10 \\
$\mathrm{Z}$ & $20 \times 4$ & 16,384 to $131,072(\times 4)$ & 1.2 to 0.15 & 10 \\
\hline
\end{tabular}

\section{References}

Bolli, P. et al. [2015] J. Astron. Instrum. 4, 150008.

Braatz, J. et al. [2010] ApJ 718, 657 .

Bragg, A. et al. [2000] ApJ 535, 73.

Comoretto, G. et al. [2006] "A modular multichannel spectrometer - design study," Arcetri Technical Report 4/2006.

Corongiu, A. [2014] "SRT antenna control: A graphic tool for setting and pointing the Sardinia radio telescope," OAC Internal Report n.40.

Davies, R. et al. [1975] MNRAS 170, 45P.

Dewey, R. et al. [1985] ApJ 294L, 25D.

Egron, E. et al. [2017] MNRAS 470, 1329.

Egron, E. et al. [2015] "Observing with the DFB at SRT: Practical notes for the user," OAC Internal Report n. 45.

Giacani, E. et al. [1997] AJ 113, 1379G.

Govoni, F., Murgia, M., Vacca, V. et al. [2017] A\&A 603A, $122 \mathrm{G}$.

Heij, V. et al. [2002] A\&A 391, 67.

Lampton, M. et al. [1992] Acta Astronaut. 26(3-4), 189.

Loi, F., Murgia, M., Govoni, F. et al. [2017] MNRAS 472, 3605L.
Lorimer, D. et al. [1995] MNRAS 273, 411.

Manchester, R. N. et al. [1972] Nat. Phys. Sci. 240, 74.

Manchester, R. N. et al. [2001] MNRAS 328, 17.

Melis, A. et al. [2014] SPIE 9153E, 2MM.

Murgia, M. et al. [2016] MNRAS 461, 3516M.

Navarrini, A. et al. [2017] URSI GASS J7-3, 2883.

Oommen, A. et al. [2014] Int. J. Innov. Res. Technol. 1(5), 800.

Orfei, A. et al. [2010] IAPM 52, 620.

Orlati, A. et al. [2012] SPIE 8451E, $2 \mathrm{MO}$.

Prandoni, I. et al. [2017] A\&A 608, A40.

Rottmann, H., Mack, K.-H., Klein, U. et al. [1996] A\&A 309, L19.

Scalambra, A. et al. [2013] "PCB FS-TP Focus Selector e Total

Power backend," IRA Internal Report n.473/13.

Spencer, A. et al. [2013] Nature 497, 224.

Tarchi, A. et al. [2018] OAC Internal Report n.72.

Valente, G. et al. [2010] SPIE 7741E, 26V.

Valente, G. et al. [2016] SPIE 9914E, 25V.

Yoshiike, S. et al. [2013] ApJ 768, 179Y. 